\title{
SONIC WELD BIODEGRADABLE PLATING SYSTEM FOR BONE GRAFT FIXATION IN ALVEOLAR CLEFT REPAIR
}

\author{
Ibrahim M. Nowair*
}

\begin{abstract}
Cleft lip and/or palate is the most widely known congenital malformation of the orofacial region. Secondary bone grafting of alveolar clefts combined with subsequent orthodontic treatment, designed to obtain a nonprosthodontic dental rehabilitation and also eliminate the other deficiencies attributable to the osseous defect. This study aimed to evaluate the role of graft fixation by using sonic weld biodegradable plating system on alveolar cleft repair
\end{abstract}

Patients and methods Ten patients with unilateral alveolar clefts ( right and left) were included in this study. The patients were evaluated with age ranging between (7-12) years. They were treated by secondary grafting procedures of their alveolar clefts using chin bone block fitted in the cleft and fixed by sonic weld biodegradable bone plates. The patients were followed up for 6 months both clinically and radiographically

Results: Uneventful soft tissue healing regarding soft tissue wounds. The alar base was augmented for all patients and the soft tissue scar had been matured (no tenderness) by about the third month postoperatively. The postoperative radiological results were excellent in all patients, regarding the alveolar bone height bordering the teeth mesially and distally, the incorporation of the graft material with the surrounding bone or the degree of eruption of the cleft related permanent teeth (lateral and canine) through the graft.

Conclusion: By the end of this study the author concluded that the fixation of monoblock corticocancellous bone graft in alveolar cleft repair using sonic weld plating system is useful as it maintain the graft volume and height.

KEY WORDS: Alveolar cleft, chin bone graft, sonic weld plating system

\section{INTRODUCTION}

The reconstruction of the continuity of the cleft alveolar process of the maxilla at recent point of view is one of the necessary conditions in reaching full rehabilitation of patients with total congenital cleft of upper lip, alveolar process of the maxilla and palate ${ }^{[1,2]}$. In the case of residual cleft of the alveolar process not only a facial aesthetics but

* Lecturer of OMFS, Faculty of Dentistry, Tanta University, EGYPT. 
also facial function, rehabilitation and integration of such patients into the society are disturbed. This is because of lack of the continuity of the dental arch, upper lip and nose deformations, speech disturbance. ${ }^{[3,4]}$.

The goals of alveolar cleft repair have both functional and aesthetic purposes the functional objectives include closure of the nasolabial fistula, creation of a stable and continuous maxillary dental arch, improved support of teeth adjacent to the cleft site, allowance for eruption of teeth into the cleft site, provision of unrestricted orthodontic movements, and facilitation of oral hygiene. With persistence of the nasolabial fistula, patients may experience chronic nasal regurgitation of liquids, resulting in chronic inflammation of the nasal mucosa, nasal discharge, and social embarrassment. ${ }^{[5]}$.

Secondary alveolar bone grafting has become a standard treatment for patients with cleft lip and palate ${ }^{[6]}$.

A few surgeons still advocate primary alveolar cleft repair with bone grafting or gingivoperiosteoplasty, maintaining that their surgical technique is different than earlier methods and that they do not observe the high incidence of midface retrusion and anterior crossbite ${ }^{[7-8]}$.

The most widely accepted time for repair protocol is during the mixed-dentition when the canine adjacent to the cleft has completed half to three quarters of its root formation ${ }^{[9]}$. Currently intermediate secondary bone grafting between 8-12 years of age is the procedure of choice for most cleft centers. There is general agreement that grafting at this time tends to improve alveolar and facial contour, and better facilitates permanent tooth eruption when compared to early secondary and tertiary grafting. ${ }^{[10-12]}$

Clinical analysis of facial growth in patients grafted at this age substantiates an absence of adverse effect on facial growth ${ }^{[13,14]}$. Furthermore, earlier repair, at the age of 5 through 7 years seems feasible and without unfavorable growth consequences. ${ }^{[15-18]}$

Fresh autogenous bone is the ideal graft because it supplies living immunocompatible bone cells essential for osteogenesis. Therefore, its transplantation is still the gold standard when harvested from sites such as iliac crest, mandible, tibia, rib, or calvarium. ${ }^{[19-20]}$

The graft fixation and stability is important for healing process as it make the resorption of graft slowly and so replacement with adequate volume of newly formed bone. Biodegradable plates and screws are used increasingly in oral and maxillofacial practice. These biodegradable plates and screws have several advantages over conventional titanium plates and screws including: no need for a second intervention to remove the devices, no interference with imaging or radiotherapeutic techniques, no possible growth disturbance and no thermal sensitivity ${ }^{21}$

A new biodegradable osteofixation system, SonicWeld has been developed. In contrast to conventional osteofixation systems, tapping of the corticalbone layer is not necessary before inserting the SonicWeld Rx biodegradable pins. A biodegradable pin is placed onto an ultrasoundactivated sonic electrode, called a sonotrode, and inserted into the bone hole. As a result of the added ultrasound energy, the thermoplastic biodegradable pin will melt, resulting in a flow of biodegradable polymers into the cortical bone layer and the cavities of the cancellous bone. There is no cellular reaction due to thermal stress during insertion. The biodegradable plate and pin head fuse at the same time. Theoretically, the fusion of plate and pinhead will result in superior mechanical device characteristics in comparison with conventional biodegradable plates ${ }^{22-24}$

So, this study was aimed to evaluate the role of graft fixation by using sonic weld biodegradable plating system on alveolar cleft repair 


\section{PATIENTS AND METHODS}

Ten patients with unilateral alveolar clefts were included in this study. They were treated by secondary grafting procedures of their alveolar clefts using chin bone block fitted in the cleft and fixed by sonic weld biodegradable bone plates. The patients were selected, examined both clinically and radiographically, and surgically managed at the Oral and Maxillofacial surgery Department, Faculty of Dentistry, Tanta University.

The patients will be selected according to the following inclusion criteria:

1) Patient's age ranged from 7-12 years

2) Unilateral alveolar cleft

3) Have no preoperative orthodontics.

4) Have no systemic disorder, which can affect the grafting outcome and healing process such as juvenile diabetes mellitus, kidney, liver, or blood diseases.

5) Have no associated craniofacial syndromes.

A biodegradable osteofixation system, SonicWeld Rx (Gebrüder Martin GmbH \& Co, Tuttlingen, Germany) was used in this study for bone graft fixation. In contrast to conventional osteofixation systems, tapping of the corticalbone layer is not necessary before inserting the SonicWeld Rx biodegradable pins. A biodegradable pin is placed onto an ultrasound-activated sonic electrode, called a sonotrode, and inserted into the bonehole. As a result of the added ultrasound energy, the thermoplastic biodegradable pin will melt, resulting in a flow of biodegradable polymers into the corticalbone layer and the cavities of the cancellous bone. There is no cellular reaction due to thermal stress during insertion. The biodegradable plate and pin head fuse at the same time. Theoretically, the fusion of plate and pinhead will result in superior mechanical device characteristics in comparison with conventional biodegradable plates ${ }^{\text {[22-24] }}$

\section{Preoperative evaluation:}

Preoperatively, all patients were examined clinically, and radio-graphically.

\section{(1) Clinical examination :}

Careful clinical examination was performed include the history, date and details of pervious surgeries for cleft lip / palate repair and chief complain.

Intraoral examination was done to determine the stability of maxillary segments, presence of old scar, asymmetry of the alar base, presence of oronasal fistula, and the presence of erupting teeth in the cleft.

\section{(2) Radiological examination:}

- It was done by using standard digital intraoral periapical and occlusal films for each patients, in which the morphology of the cleft area, the size of the cleft side, the presence or absence of permanent lateral incisor and canine, the development of root length, and stage of eruption of permanent canine and lateral incisor were also examined.

- The presence of supernumerary teeth related to the cleft site was also evaluated, and a decision was taken either to extract at least one month preoperatively if there was soft tissue deficiency or extract during surgery if enough soft tissue was available for graft coverage.

- For all patients panoramic radiograph was done to examine chin region as a donor site, regarding eruption stage of lower canines and distance in between and also for detection of any periapical pathological changes at the chin region that may affect the graft harvesting

\section{Surgical technique: figure (1\&2)}

All patients were operated under general anesthesia using nasal intubations of the normal side 


\section{The surgical technique for closure of the cleft site}

The surgical procedures for bone grafting of alveolar clefts were performed in accordance with the methods described by Tessier et al ${ }^{25}$. Through labial gingival incision, creation of labial mucoperiosteal flaps on either side of the alveolar cleft were done . the incision continues superiorly along the both side of the cleft and then scissors is used to perform the submucosal dissection superiorly to the oronasal fistula separating the muscle and connective tissue from the nasal mucosa as it extending into the base of the upper lip, the nasal mucosa is reflected palatally from the walls of the cleft. A palatal incision is then made around the teeth beginning posteriorly until the fistula site is approached at the alveolar cleft; the nasal mucosa is separated at its point of attachment to the palatal mucosa. the nasal mucosa is then completely separated from the oral mucosa of both the labial and palatal flap and from the alveolar ridge and hard palate, the separated nasal mucosa is swept back into the floor of the nose, the nasal mucosa wound edges are freshened and sutured to achieve closure with 4-0 vicryl suture material on a small cutting needle.

\section{The surgical technique for bone graft harvest}

A vestibular incision was made on the alveolar mucosa just below the attached gingiva from the second premolar region to the second premolar region in other side. The mandibular symphysis was widely exposed until the lower border of the mandible.

The volume of alveolar cleft was determined by the use of internal package paper of suture material that was trimmed and fitted to the cleft defect and then use it as a template to mark the graft size by

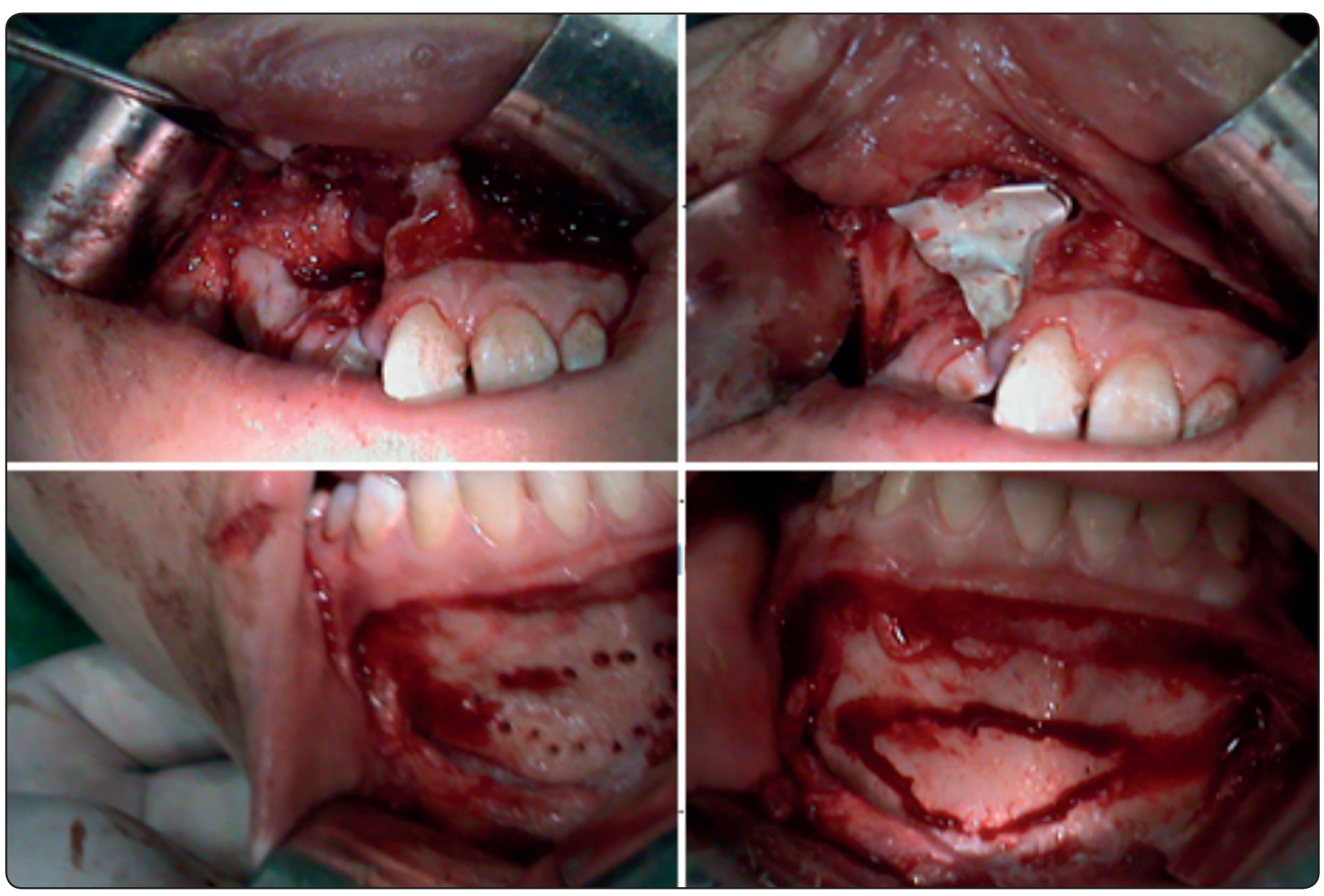

Fig. (1) Surgical steps for alveolar cleft closure and chin bone graft harvesting. 
doing multiple holes using surgical bur, then remove the paper and connect the holes to each other to be a line and complete the osteotomy by fine osteotome. A $5 \mathrm{~mm}$ safety margin below the apices and 3-5 mm thickness of the lower border was respected. After harvesting a monoblock corticocancellous bone graft, cancellous bone were harvested from the bony cavity with large curettes, the lingual cortex was left intact.

The monoblock corticocancellous bone graft was fitted in the cleft side then fixed in position using sonic weld biodegradable bone plate.

After preparation and adaptation of biodegradable plate, a pilot drill of the system described (SonicWeld Rx, KLS Martin Group, Tuttlingen, Germany) 2 holes were drilled in the labial aspect of bone in both side of the cleft. Two holes were drilled in the monoblck bone graft that was placed into the cleft site. Then a pin (SonicPin Rx, diameter $1.6 \mathrm{~mm}$, KLS Martin Group, Tuttlingen, Germany) was taken up with the sonotrode tip of the ultrasonic generator and positioned next to each drill hole for the subsequent fixation of the biodegradable plate as well as the bone graft. The ultrasonic generator was activated while in contact with the bone. This procedure changed the surface state of aggregation of the pins by melt-down from solid to liquid which allowed to easily placing the pins into the previously prepared drill holes. When the ultrasonic frequency was switched off the pins hardened immediately and firmly adhered to the local bone. The biodegradable plate and pin head fuse at the same time. Theoretically, the fusion of plate and pinhead will result in superior mechanical device characteristics in comparison with conventional biodegradable plates.

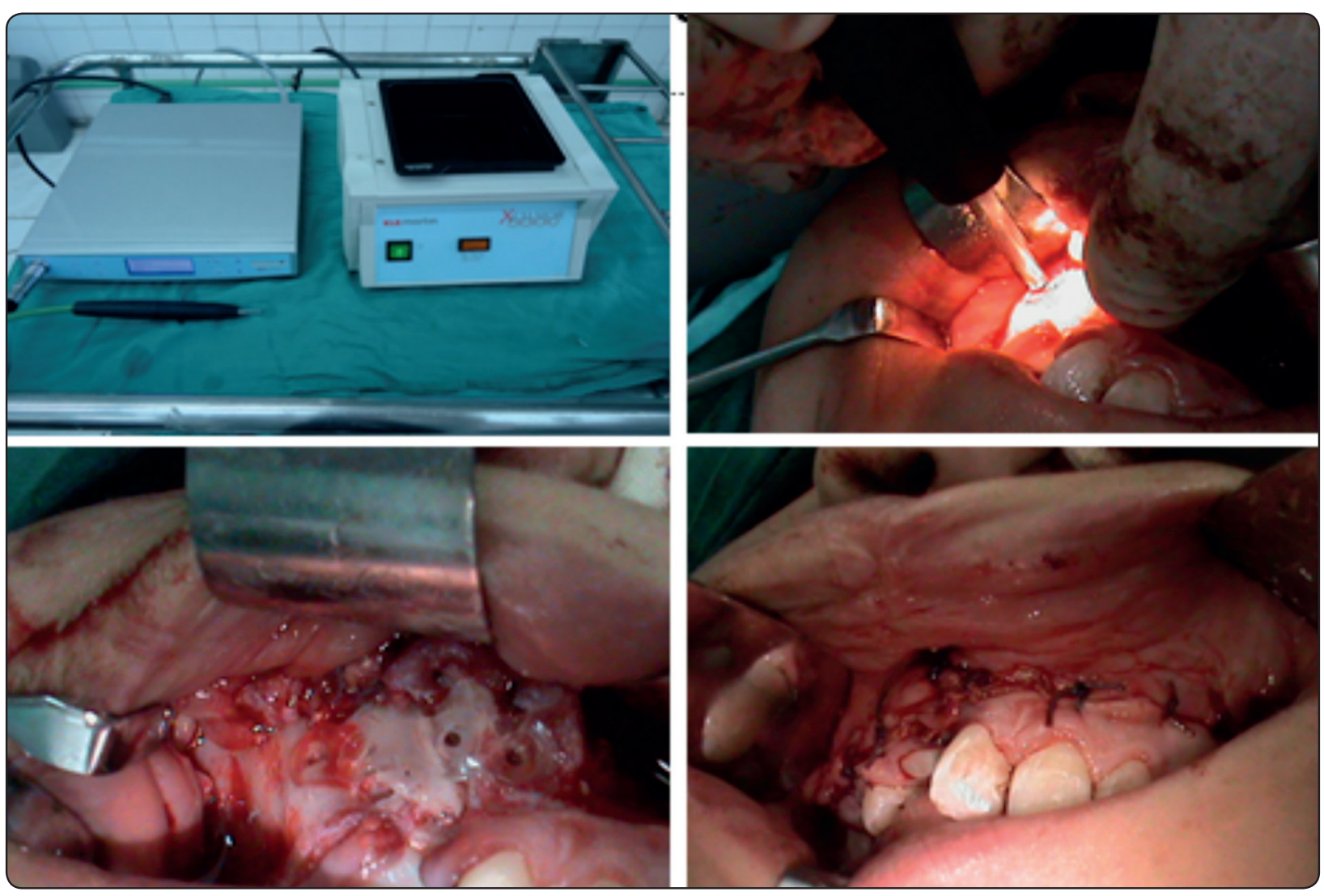

Fig. (2) Using of sonic weld and biodegradable plate for fixation of bone graft, and wound closure. 
Particles of cancellous bone were used to fill the remaining cleft defect.

The labial flaps (the sliding flaps) were advanced, and they sutured to each other and then to the palatal flap producing the classic four-corner closure over the crest of the ridge. It may be necessary to perform a small back cut or to release or score the periosteum to obtain a tension-free closure.

Postoperative administration of broad spectrum antibiotics, non-steroidal anti-inflammatory drug for pain relief and an initial dose of corticosteroids immediately postoperative to reduce postoperative edema

Soft diet was prescribed for one week, no sucking, and the need for maintaining good oral hygiene with mouth wash and brushing the remaining teeth was explained to the patients and their families.

\section{Postoperative evaluation}

All patients were followed up clinically and radiographically for 6 months

Clinically: The recipient site was evaluated for healing condition at the follow up periods ( $1^{\text {st }}$ week, $2^{\text {nd }}$ week, then $1^{\text {st }}$, and $6^{\text {th }}$ month postoperatively) regarding the presence of inflammation or infection, the soft tissue scar overlying the bone graft, tenderness of bone graft site, recurrence of oronasal fistula, stability of maxillary segments, alveolar ridge contour in cleft region, and eruption of cleft related teeth.

The donor site was evaluated regarding the presence of hematoma, parasthesia of lower lip, inflammation or infection, apical fistulae.

Radiologically: CT scan was done for estimation of the graft height outcome and eruption of cleft related teeth through the graft, at the follow up periods, determination of graft incorporation with the surrounding normal bone through the comparison of their bone density measurements using Hounsfield scale at 1 st, and 6th month postoperatively.
The donor site was examined for the presence of periapical pathology, root resorption, bone formation of the chin region at $1 \mathrm{st}$, and 6th month postoperatively.

\section{Statistical analysis}

The collected data was organized, tabulated and statistically analyzed using [software statistical computer package version 12 (SPSS)]. For quantitative data the difference between means of bone density during the follow up periods versus the preoperative one for each patient was statistically analyzed using the student ( $t$ ) test. For comparison of mean values at different periods of follow up compared to base line data, the paired t test was used. Significance was adopted at $p<0.05$ for interpretation of results of tests of significance.

\section{RESULTS}

Clinical examinations preoperatively revealed that the first chief complaint is the presence of oronasal fistula which found in all patients $(100 \%)$ which was symptomatic in $81.5 \%$ of them. The second complaint is hyper nasal speech was found in many of the patients included in this study $(81.5 \%)$, which was attributed primarily to effect of the initial cleft repair producing short palates (velopharyngeal incompetence) and secondarily due to presence of oronasal fistula.

\section{A-Recipient site (grafted site):}

\section{Clinically:}

At the first week, mild inflammation was noticed in four cases at maxillary wound and only two cases regarding the mandibular wound and did not necessitate further treatment except for continuation of the mouth wash and oral hygiene measures, local tenderness of the grafted sites was evident in all cases, there were no other signs could be detected regarding the presence of infection, flap dehiscence, graft loss or recurrence of oronasal fistula.

At the second week, normal wound healing and subsidence of all inflammation. 
At the first month and six month: Uneventful soft tissue healing regarding both maxillary and mandibular wounds.

The alar base was augmented for all patients and the soft tissue scar had been matured (no tenderness) by about the third month postoperatively.

At the end of follow up period the erupting teeth at the cleft site were in normal position and had good periodontal support.

\section{Radiographically}

Generally, the postoperative radiological results were excellent in all patients, regarding the alveolar bone height bordering the teeth mesially and distally, the incorporation of the graft material with the surrounding bone or the degree of eruption of the cleft related permanent teeth (lateral and canine) through the graft.

Quantitative computed tomography (CT) (i.e., quantitative interpretation of values derived from Hounsfield units with a suitable calibration procedure) is the modality of choice to determine bone mineral density.

- The bone density measurements of the grafted sites at the various follow up periods were illustrated in (Figure 3 and Table 1).

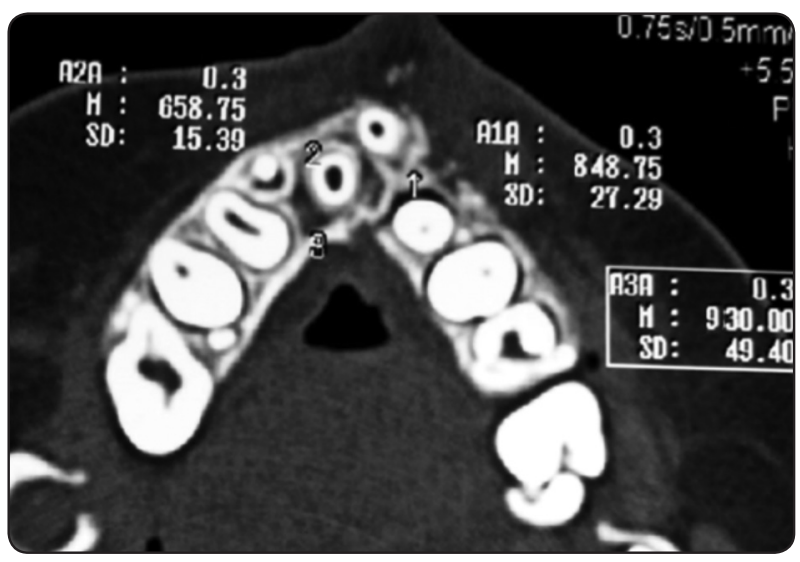

Fig. (3) Axial CT scan for alveolar grafted site after 6 months postoperative
A significant statistical difference was found between the follow up periods regarding the graft density in relation to that of the normal surrounding bone as $p$ value was 0.039

TABLE (1): Bone density measurement (from Axial CT). of the grafted sites (\% of the normal surrounding bone) at the various follow up

\begin{tabular}{|c|c|c|c|}
\hline & 1 month post & 6 month post & P Value \\
\hline Bone density & $580.4-725.5$ & $723.20-897.20$ & \multirow{2}{*}{$0.039 *$} \\
\hline Mean + SD & $652.95+102.64$ & $810.20+123.03$ & \\
\hline
\end{tabular}

* Significance

\section{DISCUSSION}

Successfully treatment of residual alveolar clefts are challenge, as such defects result from inadequate primary surgery or from a breakdown of tissue in the repaired alveolar cleft after bone grafting ${ }^{26}$. A residual alveolar cleft is an obstacle when considering rehabilitation of the dental $\operatorname{arch}^{27}$.

In this study the secondary alveolar cleft grafting technique was carried out between 7 and 12 years ago, and this is in agreement with $\mathbf{L i n} \mathbf{C}$ et $\mathbf{a l} .^{28}$, who stated that, this age is the most suitable timing for residual alveolar cleft grafting to avoid the detrimental effects on the transverse growth of the maxilla which is nearly completed by about the 6 or 7 years of age.

Establishing bony union between the clefted maxilla has well-recognized benefits, which include: preventing maxillary segmental collapse and constriction, closing of alveolar oronasal fistulas, thereby eliminating liquid regurgitation into the nose and improves speech, creation of bony support for subsequent tooth eruption and better periodontal support for teeth adjacent to the cleft, and reconstruction of the hypoplastic pyriform aperture and soft tissue of the nasal base and alar support. ${ }^{29-32}$ 
In this study the author selected the mandibular symphysis bone graft as this site has many advantages which were in agreement with SindetPedersen and Enemark ${ }^{33}$ who reported that, the use of mandibular bone grafts is increasing, because of the dense cortical structure, resorption of these bone grafts is less when compared to iliac crest, tibia, or rib bone grafts.

The use of mandibular symphysis for the following advantages over the other sites mentioned: the operations both for the cleft repair and for harvesting the graft remain confined to the oral cavity, the duration of the operation is short, the postoperative complaints are minimal, and there are no visible scares ${ }^{34,35}$

The clinical examination of the recipient sites (alveolar cleft sites) demonstrated that, all the patients included in this study had many problems related to the presence of the residual alveolar clefts. These problems were in the form of: presence of oronasal fistula (100\%) which was symptomatic in $81.5 \%$ of them. Comparable finding was reported by (Hall and Posnick ${ }^{36}$ who found that symptomatic oronasal fistula were present in $76 \%$ of the patients preoperatively. The hyper nasal speech was found in many of the patients included in this study (81.5\%), which was attributed primarily to effect of the initial cleft repair producing short palates (velopharyngeal incompetence) and secondarily to the presence of the oronasal fistula. This finding was also demonstrated by Schultes etal ${ }^{37}$ who reported leaking of air into the nose causing speech distortion due to the presence of oronasal fistula, together with the other related symptoms.

when using corticoancellous bone block graft to repair the alveolar cleft as well as support alar cartilage, it is more better to fix the graft in the recipient site to ovoid the graft movement which will affect the of graft resorption that may lead to loss of the bone volume at the grafted site. As this surgery done in young age the use of metallic hard wire not preferable because of anther surgery is needed for removal of this hardwire.
Biodegradable materials do not interfere with radio-diagnostic techniques because of their radiolucency. They promise initial strength followed by eventual degradation, resorption and elimination from the body. The good tissue acceptance enables a normal healing pattern of the bone. Avoidance of secondary implant removal operations. The use of resorbable fixation implants in developing facial bones is particulary appealing 38,39

In this study, the welding technique incorporated in the Sonicweld Rx system. A biodegradable pin is placed into an ultra-sound activated sonic electrode, called a sonotrode and inserted into the bone hole. As a result of the added ultra-sound energy, the thermoplastic biodegradable pin was melt, resulting in a flow of biodegradable polymers into the cortical bone layer and the cavities of the underlying cancellous bone. At the same time, the biodegradable plate and pinhead fuse. Due to the welding technique, the handling characteristic as well as the mechanical properties are greatly enhanced as with the studies achieved by Buijs et al., ${ }^{23}$

The analysis of the clinical and radiographic results of the current study, proved that, ultrasound based welding process by Sonic weld Rx for fixation of biodegredable plates and pins was useful in alveolar cleft graft fixation, this was in agreement with Ferguson et al., ${ }^{40}$ who stated that, the introduction of Sonic Weld with biodegradable plates will augment the mechanical properties and graft stability.

Stability as well as sufficiency of the retained graft volume to support the teeth root of an erupting or orthodontically moved tooth, closure of the preexisting oronasal fistula, in addition to the normal eruption of the related permanent teeth (lateral and canine) through the graft with healthy keratinized overlying mucosa. These clinical success criteria were advocated and determined according to Precious, ${ }^{41}$ and Tai et al. ${ }^{42}$ The postoperative radiological results were excellent in all patients, 
regarding the alveolar bone height bordering the teeth mesially and distally, the incorporation of the graft material with the surrounding bone or the degree of eruption of the cleft related permanent teeth (lateral and canine) through the graft.

Regarding the bone density in radiographic results, there was increasing in bone density at the grafted sites with progressing the follow up periods. as the means of bone density at one and six months postoperatively were $652.95( \pm 102.64)$ and 810.20 $( \pm 123.03)$ respectively. So there was a significant statistical difference between the results at one month and results at 6 months postoperatively as $\mathrm{p}$ value was 0.039 . This is in agreement with Mikołajczak et $\mathrm{al}^{43}$ who stated that relative density of the bone grafts is highly dependent on the patient's age on the day of the operation: the younger the patient is, the better mineralization of the bones is.

\section{CONCLUSION}

By the end of this study the author can concluded that the fixation of monoblock corticocancellous bone graft in alveolar cleft repair using sonic weld plating system is useful as it maintain the graft volume and height.

\section{REFERENCES}

1. Bergland O, Semb G, Abyholm FE. Elimination of the residual alveolar cleft by secondary bone grafting and subsequent orthodontic treatment. Cleft Palate J.1986; 23(3): 175-205.3.

2. Dempf R, Teltzrow T, Kramer FJ, Hausamen JE. Alveolar bone grafting in patients with complete clefts: a comparative study between secondary and tertiary bone grafting. Cleft Palate Craniofac J. 2002; 39(1): 18-25. 4.

3. Witsenburg B. The reconstruction of anterior residual bone defects in patients with cleft lip, alveolus and palate. J Maxillofac Surg. 1985; 13(5): 197-208.

4. Olekas J. and Zalecka L: Late Results of the Secondary Alveolar Bone Grafting in Complete Unilateral Cleft Lip and Palate Patients. Stomatologija, 5:17-21, 2003

5. Cohen M, Polley JW, Figueroa AA. Secondary (intermediate) alveolar bone grafting. Clin Plast Surg 1993; 20:691-705.
6. Jia YK, Fu MK, Ma L:Long-term outcome of secondary alveolar bone grafting in patients with various types of cleft. Br J Oral Maxillofac Surg 2006. 44:308-312.

7. Eppley BL. Alveolar cleft bone grafting (part I): primary bone grafting. J Oral Maxillofac Surg 1996; 54:74-82.

8. Santiago PE, Grayson BH, Cutting CB, et al. Reduced need for alveolar bone grafting by presurgical orthopedics and primary gingivoperiosteoplasty. Cleft Palate Craniofac J 1997; 35:77 -80.

9. Rocha, R., Ritter, D.E., Locks, A., de Paula, L.K. and Santana, R.M. Ideal treatment protocol for cleft lip and palate patient from mixed to permanent dentition. American Journal of Orthodontics and Dentofacial Or- thopedics, 2012,141, 140-148.

10. Bergland O, Semb G, Abyholm FE. Elimination of the residual alveolar cleft by secondary bone grafting and subsequent orthodontic treatment. Cleft Palate J. 1986;23:175205

11. Long RE Jr, Hathaway R, Daskalogiannakis J, Mercado A, Russell K, Cohen M, Semb G, Shaw W. The Americleft study: an inter-center study of treatment outcomes for patients with unilateral cleft lip and palate. Part 1. principles and study design. Cleft Palate Craniofac J. 2011;48:239243

12. Shaw WC, Dahl E, Asher-McDade C, Brattström V, Mars M, McWilliam J, Mølsted K, Plint DA, Prahl-Andersen B, Roberts C. A six-center international study of treatment outcome in patients with clefts of the lip and palate: part 5 . General discussion and conclusions. Cleft Palate Craniofac J. 1992;29:413-418..

13. Semb G. Effect of alveolar bone grafting on maxillary growth in unilateral cleft lip and palate patients. Cleft Palate $\mathrm{J} 1988 ; 25: 288-94$.

14. Daskalogiannakis J, Ross RB. Effect of alveolar bone grafting in the mixed dentition on maxillary growth in complete unilateral cleft lip and palate patients. Cleft Palate Craniofac J 1997; 34:455- 8.

15. Kortebein MJ, Nelson CL, Sadove AM. Retrospective analysis of 135 secondary alveolar cleft grafts using iliac or calvarial bone. J Oral Maxillofac Surg 1991; 49:493-8.

16. Boyne PJ. Bone grafting in the osseous reconstruction of alveolar and palatal clefts. Oral Maxillofac Surg Clin North Am 1991;3:589 - 97.

17. Hall HD, Werther JR. Conventional alveolar cleft bone grafting. Oral Maxillofac Surg Clin North Am 1991; 3: 609- 16 . 
18. Hathaway R, Daskalogiannakis J. Mercado A, Russell K, Long RE, Cohen M, Semb G, Shaw W. The Americleft study: an inter-center study of treatment outcomes for patients with unilateral cleft lip and palate. Part 2. Dental arch relationships. Cleft Palate Craniofac J. 2011;48:244-25

19. Freihofer HP, Borstlap WA, Kuijpers-Jagtmann AM, Voorsmit RA, and et al. Timing and transplant materials for closure of alveolar clefts. A clinical comparison of 296 cases. J Craniomaxillofac Surg. 1993, 21(4):143-8.

20. LaRossa D, Buchmann S, Rothkopf DM, Mayro R, Randall P. A comparison of iliac and cranial bone in secondary grafting of alveolar clefts. Plast Reconstr Surg 1995;96:789-97

21. Rokkanen PU., Bostman O., Hirvensalo E. et al: Bioabsorbable fixation in orthopaedic surgery and traumatology. Biomaterials, 2000; 21:2607.

22. Ghali GE. Sinn DP and Tantipasawasin S.: Management of nonsyndromic craniosynostosis. Atlas Oral Maxillofac Surg Clin North Am, 2002; 10:1.

23. Buijs G.J., Van der Houwen E.B., Stegenga, B., et al: Mechancal strength and stiffness of the biodegradable Sonicweld Rx osteofixation system. Int J Oral Maxillofac Surg, 2009 Apr; 67(4):782-7..

24. Pilling E., Maia R., Theissig F., Stadlinger B. et al: An experimental in vivo analysis of the resorption to ultrasound activated pins (Sonic weld ${ }^{\circledR}$ ) and standard biodegradable screws (ResorbX®) in sheep. British Journal of Oral and Maxillofacial Surgery, 2007.

25. Tessier P, Kawamoto H, Matthews D, Posnick J, Raulo Y, Tulasne JF, Wolfe SA: Autogenous bone grafts and bone substitutes--tools and techniques: A 20,000-case experience in maxillofacial and craniofacial surgery. Plast Reconstr Surg. 2005 Oct; 116(5 Suppl):6S-24S; discussion 92S-94S.

26. Horsewell B. \& Eldeeb M.: Nonporous hydroxyl apatite in the repair of alveolar clefts in a primate model. Clinical and histological findings. J. Oral Maxillofac. Surg. 1989, 47: 946.

27. .Fukuda M., Takashi T\& Yamaguchi T. et al.: Placement of endosteal implants combined with chin bone onlay graft for dental reconstruction in patients with grafted alveolar clefts. Int. J. Oral Maxillofac. Surg. 1998, 27: 440.

28. . Lin C., Lo L. \& Wang M. et al.: Major hematological disease associated with cleft lip and palate. Cleft palate Craniofac. J. 2000, 37:512.

29. Denny A., Talisman R. \& Bonawitz S.: Secondary alveolar bone grafting using milled cranial bone graft: Retrospective study of a consecutive series of 100 patients. Cleft Palate Craniofac. J. 1999, 36:144.
30. Eppley B. \& Sadove A: Management of alveolar cleft bone grafting. State of the art. Cleft palate Craniofac. J. 2000, $37: 229$.

31. Liou E., Chen P. \& Huang C. et al.: Interdental distraction osteogenesis and rapid orthodontic tooth movement: A novel approach to approximate a wide alveolar cleft or bony defect. Plast. Recnstr. Surg. 2000,105, 1262.

32. Shashua D. \& Omnell M.: Radiographic determination of the position of the maxillary lateral incisor in the cleft alveolus and parameters for assisting its habilitation prospects. Cleft palate Craniofac. J. 2000, 17:37.

33. Sindet- Pedersen S. \& Enemark H.: Mandibular bone grafts for reconstruction of alveolar clefts. J. Oral Maxillofac. Surg. 1988, 45: 533.

34. Enemark H., Jensen J. \& Bosch C.: Mandibular bone graft material for reconstruction of alveolar cleft defects: longterm results. Cleft Palate Craniofac. J2001, 38: 155.

35. Elshall M., Sadakah A and Sherif M.et al: Role of Hydroxyapatite mixed with autogenous chin bone in secondary grafting of alveolar cleft. A comparative study. DDS Thesis 2003

36. Hall $\mathrm{H}$ and Posnick J: Early results of secondary bone grafts in 106 alveolar clefts. J Oral Maxillofac Surg 1983, 41:289.

37. Schultes G., Gaggl A. \& Karcher H.: Comparison of periodontal disease in patients with clefts of palate and patients with unilateral clefts of lip, palate, and alveolus. Cleft palate Craniofac. J. 1999, 36:322.

38. Yerit K.C., Hainich S., Enislidis G. et al: Biodegradable fixation of mandibular fractures in children: stability and early results. Oral Surg Oral Med Oral Pathol Oral Radiol Endod, 2005; 100:17-24.

39. Eppley B.L.: Use of resorbable plates and screws in pediatric facial fractures. J Oral Maxillofac Surg, 2005; 63:385391.

40. Ferguson S.J., Weber U., Von Rechenberg B., et al: Enhancing the mechanical integrity of the implant-bone interface with BoneWelding $(\mathrm{R})$ technology: determination of quasi-static interfacial strength and fatigue resistance. J. Biomed. Mater. Res. B Appl. Biomate,2005; 6: 124-129

41. Precious D.: Alveolar bone grafting. J. Oral Maxillofac. Surg. Clinics North Am 2000, 12:501.

42. Tai C., Sutherland I. \& Mefadden L.: Prospective analysis of secondary alveolar bone grafting using computed tomography. J. Oral Maxillofac. Surg. 2000, 58:1241.

43. Mikołajczak T1, Wilk G, Gawrych E [Assessment of bone grafting mineralization in patients with alveolar clefts using digital radiography]. Ann Acad Med Stetin. 2007; 53(3):66-73. 